\title{
EQUIDISTRIBUTION OF FEKETE POINTS ON THE SPHERE
}

\author{
JORDI MARZO AND JOAQUIM ORTEGA-CERDÀ
}

\begin{abstract}
Fekete points are the points that maximize a Vandermonde-type determinant that appears in the polynomial Lagrange interpolation formula. They are well suited points for interpolation formulas and numerical integration. We prove the asymptotic equidistribution of Fekete points in the sphere. The way we proceed is by showing their connection to other arrays of points, the so-called Marcinkiewicz-Zygmund arrays and interpolating arrays, that have been studied recently.
\end{abstract}

\section{INTRODUCTION}

For any integer $L \geq 0$ we denote the space of spherical harmonics of degree not exceeding $L$ by $\Pi_{L}$. These vector spaces have dimensions

$$
\operatorname{dim} \Pi_{L}=\frac{d+2 L}{d}\left(\begin{array}{c}
d+L-1 \\
L
\end{array}\right)=\pi_{L} \simeq L^{d} .
$$

Let $\left\{Q_{1}^{L}, \ldots, Q_{\pi_{L}}^{L}\right\}$ be any basis in $\Pi_{L}$. The points $\mathcal{Z}(L)=\left\{z_{L, 1}, \ldots, z_{L, \pi_{L}}\right\}$ maximizing the determinant

$$
\left|\Delta\left(x_{1}, \ldots, x_{\pi_{L}}\right)\right|=\left|\operatorname{det}\left(Q_{i}^{L}\left(x_{j}\right)\right)_{i, j}\right|
$$

are called Fekete points of degree $L$ for $\mathbb{S}^{d}$ (these points are sometimes called extremal fundamental systems of points as in [10]). They are not to be confused with the elliptic Fekete points which are a system of points that minimize the potential energy. The extremal fundamental system of points are better suited nodes for cubature formulas and for polynomial interpolation, see [10] and the references therein.

The geometric properties of the distribution of Fekete points on the sphere has been the subject of research, see for instance [9], [6] or [10], where upper and lower bounds for the minimum distance between pairs of points are found. One natural problem is the limiting distribution of the points as $L \rightarrow \infty$. If we denote by $\mu_{L}=\frac{1}{\pi_{L}} \sum_{j} \delta_{x_{j}}$ it has been known long ago that for the elliptic Fekete points $\mu_{L}$ converges in the weak-* topology to the uniform distribution of the sphere even for a wide class of potentials, see [5] for a very nice survey. For Fekete points in compacts $K \subset \mathbb{C}$ this is a classical result. Much less is known in higher dimensions. In the papers $[1,2]$ the authors have found the limiting distribution in the context of line bundles over

Date: Received August 14, 2008. Accepted December, 32008.

2000 Mathematics Subject Classification. Primary 65D32; Secondary 33C55, 65T40, 11K36.

Key words and phrases. Asymptotic equidistribution, Fekete points, Marcinkiewicz-Zygmund inequalities, Interpolating arrays.

Supported by projects MTM2008-05561-C02-01, 2005SGR00611 and (the first author) NordForsk Research Network "Analysis and Applications" grant \# 080151. 
complex manifolds. Our result, Theorem 3.3, can be formally deduced from theirs but the techniques there are very different from ours, they rely on a careful study of the weighted transfinite diameter and its differentiability. We will rather emphasize the connection of Fekete points with Marcinkiewicz-Zygmund arrays and interpolating arrays (see below for the definitions). As long as the density of these arrays is understood we can obtain the equidistribution of Fekete points. This is the case of the sphere where we can build on the work [7], where MZ arrays and interpolating arrays are studied. The same approach is being pursued by Berman in line bundles over complex manifolds.

1.1. Marcinkiewicz-Zygmund inequalities and interpolation. We consider arrays of points on the sphere $\mathbb{S}^{d}$ that determine the norm of polynomials, and also arrays of points where we are free to interpolate arbitrary values by polynomials, i.e. interpolating arrays. More precisely, for any degree $L$ we take $m_{L}$ points in $\mathbb{S}^{d}$

$$
\mathcal{Z}(L)=\left\{z_{L, j} \in \mathbb{S}^{d}: 1 \leq j \leq m_{L}\right\}, \quad L \geq 0,
$$

and assume that $m_{L} \rightarrow \infty$ as $L \rightarrow \infty$. This yields a triangular array of points $\mathcal{Z}=\{\mathcal{Z}(L)\}_{L \geq 0}$ in $\mathbb{S}^{d}$.

Definition 1.1. Let $\mathcal{Z}=\{\mathcal{Z}(L)\}_{L \geq 0}$ be a triangular array with $m_{L} \geq \pi_{L}$ for all L. We call $\mathcal{Z}$ an $L^{p}$-Marcinkiewicz-Zygmund array, denoted by $L^{p}-M Z$, if there exists a constant $C_{p}>0$ such that for all $L \geq 0$ and $Q \in \Pi_{L}$,

$$
\frac{C_{p}^{-1}}{\pi_{L}} \sum_{j=1}^{m_{L}}\left|Q\left(z_{L, j}\right)\right|^{p} \leq \int_{\mathbb{S}^{d}}|Q(\omega)|^{p} d \sigma(\omega) \leq \frac{C_{p}}{\pi_{L}} \sum_{j=1}^{m_{L}}\left|Q\left(z_{L, j}\right)\right|^{p},
$$

if $1 \leq p<\infty$, and

$$
\sup _{\omega \in \mathbb{S}^{d}}|Q(\omega)| \leq C \sup _{j=1, \ldots, m_{L}}\left|Q\left(z_{L, j}\right)\right|,
$$

when $p=\infty$. Here $\sigma$ stands for the non-normalized surface area measure on the sphere $\mathbb{S}^{d}$.

In other words, the $L^{p}$-norm in $\mathbb{S}^{d}$ of a polynomial of degree $L$ is comparable to the discrete version given by the weighted $\ell^{p}$-norm of its restriction to $\mathcal{Z}(L)$.

Definition 1.2. Let $\mathcal{Z}=\{\mathcal{Z}(L)\}_{L \geq 0}$ be a triangular array with $m_{L} \leq \pi_{L}$ for all $L$. We say that $\mathcal{Z}$ is $L^{p}$-interpolating, if for all arrays $\left\{c_{L, j}\right\}_{L \geq 0,1 \leq j \leq m_{L}}$ of values such that

$$
\sup _{L \geq 0} \frac{1}{\pi_{L}} \sum_{j=1}^{m_{L}}\left|c_{L, j}\right|^{p}<\infty
$$

there exists a sequence of polynomials $Q_{L} \in \Pi_{L}$ uniformly bounded in $L^{p}$ such that $Q_{L}\left(z_{L, j}\right)=$ $c_{L, j}$, for $1 \leq j \leq m_{L}$.

Roughly speaking in order to recover the $L^{p}$-norm of a polynomial of degree $L$ from the evaluation at the points in $\mathcal{Z}(L)$ we need a sufficiently big number of points in $\mathcal{Z}(L)$. Thus, intuitively, the MZ arrays must have high density. On the other hand, in an interpolating array it is possible to have a spherical harmonic of degree at most $L$ attaining some prescribed values on $\mathcal{Z}(L)$. Intuitively this is possible only when $\mathcal{Z}(L)$ is sparse. 
In dimension one the roots of unity are simultaneously an interpolating and an $\mathrm{MZ}$ array when $1<p<\infty$. In higher dimension the situation is more delicate. It has been proved, [7, Theorem 1.7] that there are not arrays which are simultaneously $L^{p}$-MZ and interpolating when $d>2$ and $p \neq 2$ and most likely even when $p=2$. We will prove that Fekete points are a very reasonable substitute. If we perturb them slightly they are interpolating sequences and a different perturbation makes them MZ-arrays. Thus, in a sense, they behave like the roots of unity in higher dimensions. Since the densities of the MZ-arrays and the interpolating arrays are well understood, see [7, Theorem 1.6], then we will get some geometric information on Fekete points.

In the next section we will provide the connection between Fekete points and interpolating and MZ-arrays. In the last section we will draw some geometric/metric consequences.

The following notation will be used below: We write $A \lesssim B$ to denote that $A \lesssim C B$ for some constant $C>0$, independent of the degree, denoted typically by $L$. If both $A \lesssim B$ and $B \lesssim A$, then we write $A \simeq B$.

Acknowledgment This paper has its origins in a conversation of the second author with Robert Berman at the Mittag-Leffler Institute over the possibility of connecting Fekete points and sampling sequences. It is a pleasure to thank him for sharing his thoughts and to the Institute for its warm hospitality and great atmosphere.

\section{FEKETE POINTS, MZ-ARRAYS, INTERPOLATING ARRAYS}

Theorem 2.1. Given $\varepsilon>0$ let $L_{\varepsilon}=[(1+\varepsilon) L]$ and

$$
\mathcal{Z}_{\varepsilon}(L)=\mathcal{Z}\left(L_{\varepsilon}\right)=\left\{z_{L_{\varepsilon}, 1}, \ldots, z_{L_{\varepsilon}, \pi_{L_{\varepsilon}}}\right\},
$$

where $\mathcal{Z}(L)$ is the set of Fekete points of degree $L$, then $\mathcal{Z}_{\varepsilon}=\left\{\mathcal{Z}_{\varepsilon}(L)\right\}_{L \geq 0}$ is an $L^{p}-M Z$ array, for any $1 \leq p \leq \infty$.

Proof. Assume that $\mathcal{Z}$ is a collection of Fekete points. It satisfies a nice separation property that is convenient to prove the first inequality of (1.1).

Definition 2.2. A triangular array $\mathcal{Z}$ is uniformly separated if there is a positive number $\varepsilon>0$ such that

$$
d\left(z_{L, j}, z_{L, k}\right) \geq \frac{\varepsilon}{L+1} \text {, if } j \neq k
$$

for all $L \geq 0$, where $d(z, w)=\arccos \langle z, w\rangle$.

Reimer observed that Fekete points are uniformly separated, [9, p. 199]. More precisely, he proves that

$$
\frac{\pi}{2 L} \leq \min _{i \neq j} d\left(z_{L, i}, z_{L, j}\right)
$$

using the analogous result of M. Riesz for trigonometric polynomials on great circles. Thus we know that

$$
\min _{i \neq j} d\left(z_{L_{\varepsilon}, i}, z_{L_{\varepsilon}, j}\right) \geq \frac{\pi}{2 L_{\varepsilon}} \geq \frac{C_{\varepsilon}}{L+1},
$$


and therefore the array $\mathcal{Z}_{\varepsilon}$ is uniformly separated. This implies the following Plancherel-Polya type inequality for any $1 \leq p<\infty$

$$
\frac{1}{\pi_{L}} \sum_{j=1}^{\pi_{L_{\varepsilon}}}\left|Q\left(z_{L_{\varepsilon}, j}\right)\right|^{p} \lesssim \int_{\mathbb{S}^{d}}|Q(z)|^{p} d \sigma(z), \text { for any } Q \in \Pi_{L},
$$

see [7, Corollary 4.6].

The right hand side inequality in (1) is more delicate, we need an appropriate representation formula for the polynomials in terms of the values at the points. The most naive approach is to start by the Lagrange interpolation formula. Let

$$
\ell_{L, i}(z)=\frac{\Delta\left(z_{L, 1}, \ldots, z_{L, i-1}, z, z_{L, i+1}, \ldots, z_{L, \pi_{L}}\right)}{\Delta\left(z_{L, 1}, \ldots, z_{L, \pi_{L}}\right)}
$$

then $\sup _{z \in \mathbb{S}^{d}}\left|\ell_{L, i}(z)\right| \leq 1$ and the Lagrange interpolation operator defined in $\mathcal{C}\left(\mathbb{S}^{d}\right)$ as

$$
\Lambda_{L}(f)(z)=\sum_{j=1}^{\pi_{L}} f\left(z_{L, j}\right) \ell_{L, j}(z)
$$

satisfies

$$
\left\|\Lambda_{L}(f)\right\|_{\infty} \leq \pi_{L}\|f\|_{\infty} .
$$

We want better control of the norms. So we need a slightly bigger set of points and a weighted representation formula. Let $p$ be a polynomial in one variable of degree $[L \varepsilon]$ and such that $p(1)=1$. Then given $Q \in \Pi_{L}$ one has for a fixed $z \in \mathbb{S}^{d}$

$$
R(w)=Q(w) p(\langle z, w\rangle) \in \Pi_{L_{\varepsilon}}
$$

and therefore we obtain our weighted representation formula:

$$
Q(z)=\sum_{j=1}^{\pi_{L_{\varepsilon}}} p\left(\left\langle z, z_{L_{\varepsilon}, j}\right\rangle\right) Q\left(z_{L_{\varepsilon}, j}\right) \ell_{L_{\varepsilon}, j}(z) .
$$

We define the operator $Q_{L}$ from $\mathbb{C}^{\pi_{L_{\varepsilon}}} \rightarrow \Pi_{L_{2 \varepsilon}}$ as

$$
Q_{L}[v](z)=\sum_{j=1}^{\pi_{L_{\varepsilon}}} v_{j} p\left(\left\langle z, z_{L_{\varepsilon}, j}\right\rangle\right) \ell_{L_{\varepsilon}, j}(z) \quad \forall v \in \mathbb{C}^{\pi_{L_{\varepsilon}}} .
$$

We want to prove that

$$
\int_{\mathbb{S}^{d}}\left|Q_{L}[v](z)\right|^{p} d \sigma(z) \lesssim \frac{1}{\pi_{L_{\varepsilon}}} \sum_{j=1}^{\pi_{L_{\varepsilon}}}\left|v_{j}\right|^{p},
$$

with constants uniform in $L$ which is the right-hand side of (1). We need to choose the weight $p$ with care. We need a polynomial $p$ that peaks at one point, has degree $[\varepsilon L]$ and decays fast far away from the peak point. For this purpose we will use powers of the Jacobi polynomials which are natural in this context because they are the reproducing kernels in $\Pi_{L}$, see [7]. The Jacobi 
polynomials $P_{L}^{(\alpha, \beta)}$ of degree $L$ and index $(\alpha, \beta)$ are the orthogonal polynomials on [-1, 1] with respect to the weight function $(1-x)^{\alpha}(1+x)^{\beta}$ with $\alpha, \beta>-1$. We take the normalization

$$
P_{L}^{(\alpha, \beta)}(1)=\left(\begin{array}{c}
L+\alpha \\
L
\end{array}\right) \simeq L^{\alpha} .
$$

We can use the estimates in [11, Section 7.34] to obtain, for any $v \in \mathbb{S}^{d}$

$$
\int_{\mathbb{S}^{d}}\left|P_{L}^{(d / 2, d / 2-1)}(\langle u, v\rangle)\right|^{2} d \sigma(u) \simeq 1, \quad \forall L>0 .
$$

We will use as auxiliary polynomial

$$
p(t)=L^{-d}\left(P_{[\varepsilon L / 2]}^{(d / 2, d / 2-1)}(t)\right)^{2},
$$

then $p(1) \simeq 1$ and by the estimate (3)

$$
\int_{\mathbb{S}^{d}}\left|p\left(\left\langle z, z_{L_{\varepsilon}, j}\right\rangle\right)\right| d \sigma(z) \simeq L^{-d} \int_{\mathbb{S}^{d}}\left|P_{[\varepsilon L / 2]}^{(d / 2, d / 2-1)}\left(\left\langle z, z_{L_{\varepsilon}, j}\right\rangle\right)\right|^{2} d \sigma(z) \simeq L^{-d} \simeq \pi_{L}^{-1} .
$$

Now as $\left|\ell_{L_{\varepsilon}, j}(z)\right| \leq 1$ one has

$$
\int_{\mathbb{S}^{d}}\left|Q_{L}[v]\right| d \sigma(z) \leq \sum_{j=1}^{\pi_{L_{\varepsilon}}}\left|v_{j}\right| \int_{\mathbb{S}^{d}}\left|p\left(\left\langle z, z_{L_{\varepsilon}, j}\right\rangle\right)\right| d \sigma(z) \lesssim \frac{1}{\pi_{L}} \sum_{j=1}^{\pi_{L_{\varepsilon}}}\left|v_{j}\right|
$$

and also for any fixed $z \in \mathbb{S}^{d}$

$$
\begin{aligned}
\left|Q_{L}[v](z)\right| & \leq \sup _{j}\left|v_{j}\right| \sum_{j=1}^{\pi_{L_{\varepsilon}}}\left|p\left(\left\langle z, z_{L_{\varepsilon}, j}\right\rangle\right)\right| \\
& \lesssim \sup _{j}\left|v_{j}\right| \int_{\mathbb{S}^{d}} \pi_{L}\left|p\left(\left\langle z, z_{L_{\varepsilon}, j}\right\rangle\right)\right| d \sigma(z) \lesssim \sup _{j}\left|v_{j}\right| .
\end{aligned}
$$

Then the result follows by the Riesz-Thorin interpolation theorem.

The corresponding result for interpolation reads as follows:

Theorem 2.3. Given $\varepsilon>0$ let $L_{-\varepsilon}=[(1-\varepsilon) L]$ and let

$$
\mathcal{Z}_{-\varepsilon}(L)=\mathcal{Z}\left(L_{-\varepsilon}\right)=\left\{z_{L_{-\varepsilon}, 1}, \ldots, z_{L_{-\varepsilon}, \pi_{L_{-\varepsilon}}}\right\}
$$

where $\mathcal{Z}(L)$ is a set of Fekete points of degree $L$, then the array $\mathcal{Z}_{-\varepsilon}=\left\{\mathcal{Z}_{-\varepsilon}(L)\right\}_{L \geq 0}$ is $L^{p}$ interpolating, for any $1 \leq p \leq \infty$.

Proof. Given an array of values $\left\{v_{L_{-\varepsilon}, j}\right\}_{j=1}^{\pi_{L_{-\varepsilon}}}$, we can define the polynomials in $\Pi_{L}$

$$
R_{L}[v](z)=\sum_{j=1}^{\pi_{L_{-\varepsilon}}} v_{L_{-\varepsilon} j} p\left(\left\langle z, z_{L_{-\varepsilon}, j}\right\rangle\right) \ell_{L_{-\varepsilon}, j}(z)
$$

and $R_{L}\left(z_{L_{-\varepsilon}, j}\right)=v_{L_{-\varepsilon}, j}$. This time the map $R_{L}$ is from $\mathbb{C}^{\pi_{L_{-\varepsilon}}} \rightarrow \Pi_{L}$ and the $L^{p}$-estimates on the norm of $R_{L}$ follow exactly as the estimates of $Q_{L}$ in the previous theorem. 


\section{GEOMETRIC PROPERTIES OF FEKETE POINTS}

We will draw some geometric information on Fekete points. For a given $z \in \mathbb{S}^{d}$ and $0<R<1$ we denote by $B(z, R)$ the spherical cap $B(z, R)=\left\{w \in \mathbb{S}^{d} ; d(z, w)<R\right\}$. We will prove that as $L \rightarrow \infty$ the number of Fekete points in $B(z, R)$ gets closer to $\pi_{L} \tilde{\sigma}(B(z, R))$ where $\tilde{\sigma}$ is the normalized Lebesgue measure on $\mathbb{S}^{d}$, i.e. $\tilde{\sigma}=\sigma / \sigma\left(\mathbb{S}^{d}\right)$. We need first information on the density of $\mathrm{MZ}$ and interpolation arrays.

Definition 3.1. For $\mathcal{Z}$ a uniformly separated triangular array in $\mathbb{S}^{d}$ we define the upper and lower density of the array respectively as

$$
\begin{gathered}
D^{-}(\mathcal{Z})=\liminf _{\alpha \rightarrow \infty} \liminf _{L \rightarrow \infty} \frac{\min _{z \in \mathbb{S}^{d}} \#(\mathcal{Z}(L) \cap B(z, \alpha / L)) / \pi_{L}}{\tilde{\sigma}(B(z, \alpha / L))}, \\
D^{+}(\mathcal{Z})=\limsup _{\alpha \rightarrow \infty} \limsup _{L \rightarrow \infty} \frac{\min _{z \in \mathbb{S}^{d}} \#(\mathcal{Z}(L) \cap B(z, \alpha / L)) / \pi_{L}}{\tilde{\sigma}(B(z, \alpha / L))} .
\end{gathered}
$$

The main result in [7, Theorem 1.6] is (with a slight different notation).

Theorem 3.2. Let $1 \leq p \leq \infty$. Let $\mathcal{Z}$ be a uniformly separated array. If $\mathcal{Z}$ is an $L^{p_{-}}$ Marcinkiewicz-Zygmund array then $D^{-}(\mathcal{Z}) \geq 1$. On the other hand if $\mathcal{Z}$ is an $L^{p}$-interpolating array then $D^{+}(\mathcal{Z}) \leq 1$.

From this result and the two theorems in the previous section we will deduce the asymptotic equidistribution of Fekete points on $\mathbb{S}^{d}$.

Theorem 3.3. Let $\mathcal{Z}=\{\mathcal{Z}(L)\}_{L \geq 0}$ be any array such that $\mathcal{Z}(L)$ is a set of Fekete points of degree $L$ and $\mu_{L}=\frac{1}{\pi_{L}} \sum_{j=1}^{\pi_{L}} \delta_{z_{L, j}}$, then $\mu_{L}$ converges in the weak-* topology to the normalized surface area measure on $\mathbb{S}^{d}$.

Proof. We know that for any $\varepsilon>0$ the array $\mathcal{Z}_{\varepsilon}=\left\{\mathcal{Z}_{\varepsilon}(L)\right\}_{L \geq 0}$ is $L^{2}$-MZ, so if we unwind the definitions corresponding to the densities, we get that for any $\varepsilon>0$, there is a big $\alpha=\alpha(\varepsilon)$ such that for all $L$ and $z \in \mathbb{S}^{d}$

$$
\frac{\frac{1}{\pi_{L}} \#\left(\mathcal{Z}(L) \cap B\left(z, \frac{\alpha}{L}\right)\right)}{\tilde{\sigma}\left(B\left(z, \frac{\alpha}{L}\right)\right)} \geq(1-\varepsilon) .
$$

Similarly since $\mathcal{Z}_{-\varepsilon}$ is interpolating, whenever $\mathcal{Z}$ is a Fekete array, from the density condition we get that there is a big $\alpha=\alpha(\varepsilon)$ such that for all $L$ and $z \in \mathbb{S}^{d}$

$$
\frac{\frac{1}{\pi_{L}} \#\left(\mathcal{Z}(L) \cap B\left(z, \frac{\alpha}{L}\right)\right)}{\tilde{\sigma}\left(B\left(z, \frac{\alpha}{L}\right)\right)} \leq(1+\varepsilon) \text {. }
$$

We have

$$
\begin{aligned}
\left(\mu_{L} * \chi_{B(N, \alpha / L)}\right)(z) & =\int_{\nu \in S O(d+1)} \chi_{B(N, \alpha / L)}\left(\nu^{-1} z\right) \mu_{L}(\nu N) d \nu \\
& =\frac{1}{\pi_{L}} \#(\mathcal{Z}(L) \cap B(z, \alpha / L))
\end{aligned}
$$

and

$$
\left(\sigma * \chi_{B(N, \alpha / L)}\right)(z)=\sigma(B(z, \alpha / L))
$$


where $N=(0, \ldots, 0,1)$ stands for the north pole of $\mathbb{S}^{d}$. So finally for any $\varepsilon>0$ there is a big $\alpha$ such that

$$
(1-\varepsilon)\left(\tilde{\sigma} * \chi_{B(N, \alpha / L)}\right)(z) \leq\left(\mu_{L} * \chi_{B(N, \alpha / L)}\right)(z) \leq(1+\varepsilon)\left(\tilde{\sigma} * \chi_{B(N, \alpha / L)}\right)(z),
$$

for any $z$ and $L \geq 1$.

We take an arbitrary spherical cap $B(z, r)$. We want to check that $\mu_{L}(B(z, r)) \rightarrow \tilde{\sigma}(B(z, r))$ as $L \rightarrow \infty$. We fix an $\varepsilon>0$ and we take the convolution of (4) with the function $\frac{\chi_{B(N, r)}}{\tilde{\sigma}(B(N, \alpha / L))}$ with a very big $L$ and this proves

$$
(1-\varepsilon)\left(\tilde{\sigma} * \chi_{B(N, r-\alpha / L)}\right)(z) \leq\left(\mu_{L} * \chi_{B(N, r+\alpha / L)}\right)(z)
$$

and

$$
\left(\mu_{L} * \chi_{B(N, r-\alpha / L)}\right)(z) \leq(1+\varepsilon)\left(\tilde{\sigma} * \chi_{B(N, r+\alpha / L)}\right)(z),
$$

for any $z$ and $L$ big. We take limits as $L \rightarrow \infty$ and since $\mathcal{Z}$ is uniformly separated that means that $\mu_{L}(B(z, r+\alpha / L) \backslash B(z, r-\alpha / L)) \rightarrow 0$ uniformly in $z \in \mathbb{S}^{d}$ as $L \rightarrow \infty$. Thus

$$
\lim _{L \rightarrow \infty} \mu_{L}(B(z, r))=\tilde{\sigma}(B(z, r))
$$

for an arbitrary spherical cap. This already implies the convergence in the weak-* topology of the measures, see [3], i.e.

$$
\lim _{L \rightarrow \infty} \frac{1}{\pi_{L}} \sum_{j=1}^{\pi_{L}} f\left(z_{L, j}\right)=\frac{1}{\sigma\left(\mathbb{S}^{d}\right)} \int_{\mathbb{S}^{d}} f(z) d \sigma(z)
$$

for any $f \in \mathcal{C}\left(\mathbb{S}^{d}\right)$.

From the uniform density condition on Fekete points we may obtain other geometric consequences on the distribution of Fekete points $\mathcal{Z}(L)$. We give one example. It is well known that using the bound given by Fejes Tóth [4] for the maximum of the minimal spherical distance between any set of $\pi_{L}=(L+1)^{2}$ points on $\mathbb{S}^{2}$, there exist at least two points in $\mathcal{Z}(L)$ at distance $d_{L}$ with

$$
d_{L} \leq \arccos \frac{\cot ^{2} \omega_{L}-1}{2}, \quad \omega_{L}=\frac{(L+1)^{2}}{(L+1)^{2}-2} \frac{\pi}{6}
$$

but as

$$
L \arccos \frac{\cot ^{2} \omega_{L}-1}{2} \nearrow \kappa=3.80925 \ldots, \quad \text { when } L \rightarrow \infty
$$

one has

$$
\min _{i \neq j} d\left(z_{L, i}, z_{L, j}\right) \leq \frac{\kappa}{L} .
$$

However the numerical results in [10, p. 122] suggest that the right asymptotic should be

$$
\lim _{L \rightarrow \infty} L \min _{i \neq j} d\left(z_{L, i}, z_{L, j}\right)=\pi .
$$

To bound the maximal number, $\mathcal{N}$, of disjoint spherical caps in $\mathbb{S}^{2}$ of radius $\eta / L$ to be found in a larger spherical cap of radius $\alpha / L$ we use the following result due to Molnár

$$
\mathcal{N} \leq \frac{\pi}{\sqrt{12}} \frac{\sigma\left(B\left(z, \frac{\alpha}{L}\right)\right)}{\sigma\left(B\left(z, \frac{\eta}{2 L}\right)\right)}
$$


see [8]. Then substituting in the density condition one gets

$$
\eta \leq 4 \sqrt{\frac{\pi}{\sqrt{12}}}=\kappa .
$$

So, we get not only that there exists a pair of points in each generation $\mathcal{Z}(L)$ at distance smaller than $\kappa / L$ but a more uniform estimate: for any $\varepsilon>0$ there is an $\alpha$ such that:

$$
\inf _{z_{L, i}, z_{L, j} \in B(z, \alpha / L)} d\left(z_{L, i}, z_{L, j}\right) \leq \frac{\kappa+\varepsilon}{L}
$$

for any spherical cap $B(z, \alpha / L)$.

Up to now we have drawn information from MZ-arrays and interpolating arrays to get new information on Fekete points, but the reverse direction can also be useful. For instance, since any Fekete array has density one and a small perturbation makes it a MZ-array (or interpolation), then we obtain the following corollary

Corollary 3.4. Given any $\epsilon>0$, there are arrays $\mathcal{Z}_{\varepsilon}$ and $\mathcal{Z}_{-\varepsilon}$ with densities $D^{+}\left(\mathcal{Z}_{\varepsilon}\right)=$ $D^{-}\left(\mathcal{Z}_{\varepsilon}\right)=1+\varepsilon, D^{+}\left(\mathcal{Z}_{-\varepsilon}\right)=D^{-}\left(\mathcal{Z}_{-\varepsilon}\right)=1-\varepsilon$, such that $\mathcal{Z}_{\varepsilon}$ is an $L^{p}-M Z$ array for any $p \in[1, \infty]$ and $\mathcal{Z}_{-\varepsilon}$ is an $L^{p}$-interpolating array for any $p \in[1, \infty]$.

Thus the necessary density conditions that MZ-arrays and interpolating arrays satisfy are sharp.

\section{REFERENCES}

[1] R. BERMAN, S. BOUCKSOM (2008): Equidistribution of Fekete points on complex manifolds, arxiv:0807.0035 [math.CV].

[2] R. BERMAN, S. BOUCKSOM, D. W. NYSTRÖM (2008): Convergence towards equilibrium on complex manifolds, Oberwolfach Report No. 38, 7-12.

[3] M. BLÜMLINGER (1990): Asymptotic distribution and weak convergence on compact Riemannian manifolds, Monatsh. Math. 110, no. 3-4, 177-188. MR MR1084310 (92h:58033)

[4] L. FEJES TÓTH (1949): On the densest packing of spherical caps, Amer. Math. Monthly 56, 330-331. MR MR0030217 (10,731b)

[5] D. P. HARDIN, E. B. SAFF (2004): Discretizing manifolds via minimum energy points, Notices Amer. Math. Soc. 51, no. 10, 1186-1194. MR MR2104914 (2006a:41049)

[6] L. BOS, N. LEVENBERG, S. WALDRON (2008): On the spacing of Fekete points for a sphere, ball or simplex, To appear in Indag. Math.

[7] J. MARZO (2007): Marcinkiewicz-Zygmund inequalities and interpolation by spherical harmonics, J. Funct. Anal. 250, no. 2, 559-587. MR MR2352491

[8] J. MOLNÁR (1952): Ausfüllung und Überdeckung eines konvexen sphärischen Gebietes durch Kreise. I, Publ. Math. Debrecen 2, 266-275. MR MR0053539 (14,788a)

[9] M. REIMER (1990): Constructive theory of multivariate functions, Bibliographisches Institut, Mannheim, With an application to tomography. MR MR1115901 (92m:41003)

[10] I. H. SLOAN, R. S. WOMERSLEY (2004): Extremal systems of points and numerical integration on the sphere, Adv. Comput. Math. 21, no. 1-2, 107-125. MR MR2065291 (2005b:65024)

[11] G. SZEGÖ (1975): Orthogonal polynomials, fourth ed., American Mathematical Society, Providence, R.I., American Mathematical Society, Colloquium Publications, Vol. XXIII. MR MR0372517 (51 \#8724) 
DEPARTMENT OF MATHEMATICAL SCIENCES

Norwegian University of SCIEnCE AND TeChnology, N-7491 TROndheim, Norway

E-mail address: jordi.marzo@math.ntnu.no

DEPARTAMENT DE MATEMÀTICA APPLICADA I ANÀLISI

UNIVERSITAT DE BARCELONA, GRAN Via 585, 08007-BARCELONA, SPAin

E-mail address: jortega@ub. edu 